\title{
Moving Mass and Reaction Compound Control System Design for Reentry Aircrafts
}

\author{
Jun Zhou ${ }^{1}$, Xiaoting Wang ${ }^{1} \&$ Peng Lin ${ }^{1}$ \\ ${ }^{1}$ Institute of Precise Guidance and Control, Northwestern Polytechnical University, Xi'an, China \\ Correspondence: Jun Zhou, Institute of Precise Guidance and Control, Northwestern Polytechnical University, \\ Xi'an 710072, China. E-mail: loperman@yahoo.cn
}

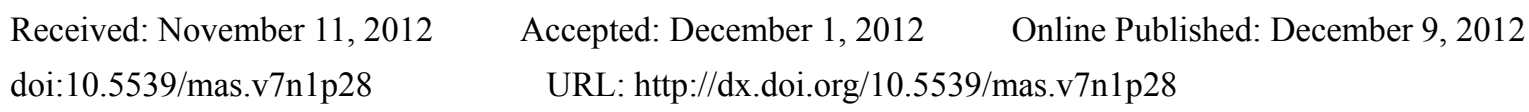

\begin{abstract}
Moving mass control (MMC) method is of benefit to reentry aircrafts in shape maintenance, energy consume reduction and load capability increase. However, as an aerodynamic manipulating method, MMC partially lacks ability to perform effectively during a whole airspace flight. To achieve reentry aircrafts control system design goal of high performance and low energy cost, we propose a moving mass and reaction compound control system design method in this paper. Firstly, dynamic models are established for a reentry aircraft actuated by moving mass and reaction compound control system, including MMC actuator models. Secondly, moving mass control periodic equivalent torque (MMCPET) is defined, and an optimal torque distribution compound control method based on MMCPET prediction is presented. The optimal object of torque distribution is minimizing difference from MMCPET and control torque command given by virtual controller. Finally, comparison simulations are performed to demonstrate validity of the proposed method. Simulation results show that the proposed compound control is more effective than moving mass control. Also, even if performance of compound control is nearly the same as that of reaction control, thrust impulse requirement of the former is close to $1 / 5$ of the latter.
\end{abstract}

Keywords: compound control, moving mass control $(\mathrm{mmc})$, reaction control, optimal torque distribution

\section{Introduction}

As a special attitude control method for reentry aircrafts, Moving Mass Control (MMC) has distinct advantages from rudder control and reaction control. For example, the servo system of MMC is settled inside the shell, which makes the control performance of MMC more reliable than rudder control. Also, by using all aerodynamic forces acting on the shell to provide control torque, MMC is more effective than rudder control and need less energy cost than reaction control. Unfortunately, as an aerodynamic manipulating method, MMC is not always able to provide high performance attitude control for a whole airspace reentry aircraft.

As we all known, reaction control system (RCS) is able to provide stable control torque in any aerodynamic condition. Therefore, combining moving mass control with reaction control, we can obtain a new control method that will lead to whole airspace high attitude control performance of reentry aircrafts. According to KONG Xue et al. (2009), adjusting static stability by MMC is helpful to improve reaction control performance. However, the effect of MMC torque on attitude control is not focused in her research, nor is to make full use of MMC torque in compound control system design for reducing energy cost.

In this paper, we focus on making full use of MMCS control torque in moving mass and reaction compound control system design, in order to obtain a compound control system that is high performance and low energy cost, and an optimal torque distribution compound control method is proposed.

\section{Dynamic Models}

\subsection{Compound Control System Collectivity}

Consider an axial symmetry reentry aircraft actuated by both moving mass control system and reaction control system. The former control system includes three one-dimensional movable blocks. Each of them can move along one of body frame axes without any rotation relative to the body frame. As for the latter, twelve pulse-width modulated thrusters compose the reaction control system. Thrusters for pitch and yaw are located in pairs in the nose and tail of the aircraft, while those for roll are located in the middle. All thrusters are configured 
to allow "coupled" RCS firings (where thrusters on opposite sides of the aircraft fired together), which allows adjusting the aircrafts attitude without affecting the critical accuracy of their trajectories.

Considering that the reentry aircraft is axial symmetry, the compound control system collectivity for only pitch channel is shown in Figure 1. Here, $\mathrm{P}$ represents press center, $\mathrm{C}$ represents the total mass center, and $\mathrm{O}$ represents mass center of the shell, which is the aircraft except for moving mass control system. Let $\boldsymbol{r}_{\boldsymbol{O A}_{1}}^{\boldsymbol{B}}=\left[\begin{array}{lll}\delta_{1} & 0 & 0\end{array}\right]^{T}, \boldsymbol{r}_{\boldsymbol{O A}_{2}}^{\boldsymbol{B}}=\left[\begin{array}{lll}l_{2} & \delta_{2} & 0\end{array}\right]^{T}, \boldsymbol{r}_{\boldsymbol{O A}_{3}}^{\boldsymbol{B}}=\left[\begin{array}{lll}l_{3} & 0 & \delta_{3}\end{array}\right]^{T}$ be separately the position vector of movable blocks with respect to point $\mathrm{O}$ written in body frame, where $\delta_{i}, i=1,2,3$ are constrained variable coordinates, and $l_{2}, l_{3}$ are constant axial offsets. Let $\boldsymbol{R}^{B}=\left[\begin{array}{lll}R_{x} & R_{y} & R_{z}\end{array}\right]^{T}$ be aerodynamic force vector written in body frame, $F$ be the reaction thrust, and $L_{p z}$ be the distance between two coupled pitch thrusters.

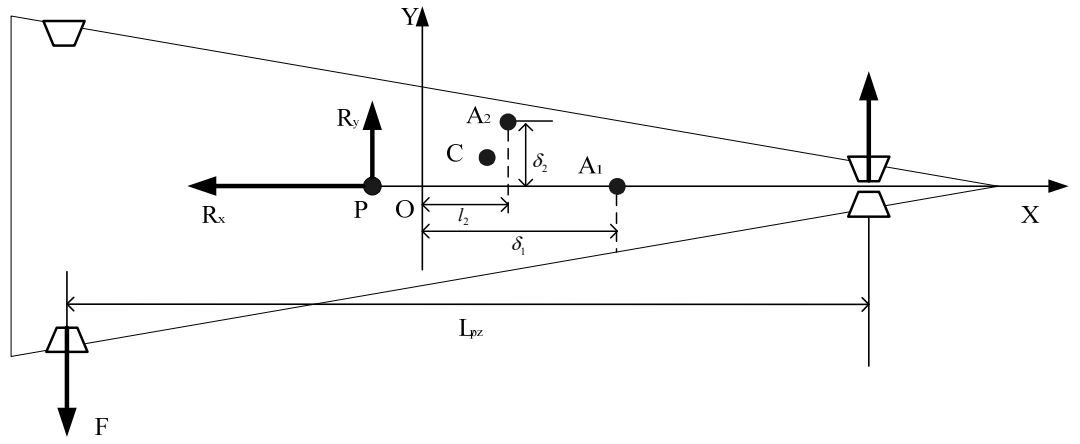

Figure 1. Compound control system collectivity for pitch channel

\subsection{Govern Equation of Attitude Motion}

Based on angular momentum theorem, vector equation of attitude motion for a reentry aircraft actuated by moving mass and reaction compound control system, which is written in body frame, can be described as

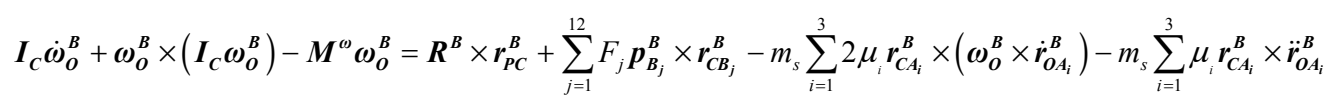

Where, $\boldsymbol{\omega}_{\boldsymbol{o}}^{B}$ is angular velocity, $\boldsymbol{M}^{\boldsymbol{\omega}} \in R^{3 \times 3}$ is aerodynamic damping, $m_{s}$ is total mass, $\boldsymbol{I}_{C}$ is inertia and $\boldsymbol{r}_{P C}^{B}$ is the vector from point $\mathrm{P}$ to point $\mathrm{C}$. For the ith movable block, $\mu_{i}$ is the ratio from block mass to total mass, and $\dot{\boldsymbol{r}}_{O A_{i}}^{B}, \ddot{r}_{O A_{i}}^{B}$ are block velocity and acceleration with respect to body frame, $\boldsymbol{r}_{C A_{i}}^{B}$ is the block position vector with respect to point C. For the jth thruster, $F_{j}$ is the thrust value, $\boldsymbol{p}_{B_{j}}^{B}$ is the direction vector, and $\boldsymbol{r}_{C B_{j}}^{\boldsymbol{B}}$ is the vector from point $\mathrm{C}$ to the point of thrust.

\subsection{Actuator Models of MMCS}

The servo characteristic of the movable block can be described as a second order object, therefore motion models of MMCS actuators are

$$
\ddot{\delta}_{i}=-2 \xi_{i} \omega_{i} \dot{\delta}_{i}-\omega_{i}^{2} \delta_{i}+\omega_{i}^{2} \delta_{i c}, \quad i=1,2,3
$$

Where, $\delta_{i c}$ is coordinate command of the ith block given by controller, and $\xi_{i}, \omega_{i}$ are relative servo damping and nature frequency. Coordinate commands are restricted by space constrains of the blocks.

\section{Optimal Torque Distribution Compound Control Method}

To make up for MMC deficiency, reaction control is introduced to form a compound control method of whole airspace reentry aircrafts. Considering characteristics of those control systems, any good design of the compound control should aim at high performance and low energy cost. One simple design to achieve that goal is dynamically distributing control torque demands given by virtual controller, in the way of making MMC torque as close to torque demands as possible. However, MMC is a continuous control method while reaction control is discrete, which makes the compound system a hybrid system. Consequently, it is difficult to design torque distribution rules without uniform criterion to evaluate control torques provided by MMCS and RCS.

In this paper, we choose periodic equivalent torque to be the uniform torque evaluation criterion, and propose an optimal torque distribution compound control method based on MMC periodic equivalent prediction. Compound control design for only pitch channel is given as an example, and following problems are solved:

- What is MMC periodic equivalent torque (MMCPET) and how to calculate or even predict it;

- What is the optimization function to make full use of MMC torque in the compound control design; 
- How to solve the optimal problem and obtain servo commands for both MMCS and RCS.

The structure of proposed compound control is shown in Figure 2.

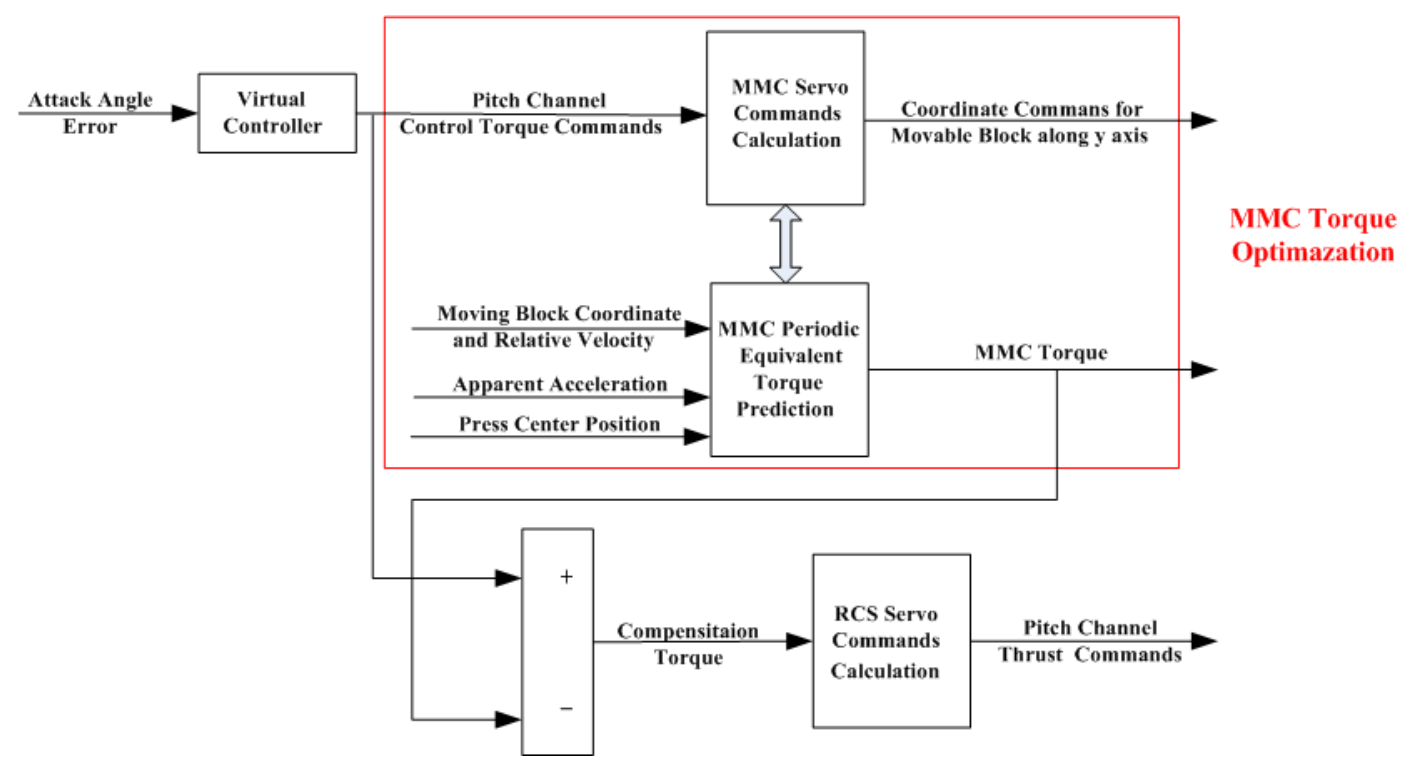

Figure 2. Optimal torque distribution compound control structure

\subsection{Virtual Controller Design}

Suppose lateral motion is indistinctive, attitude motion equations for pitch channel, derived from Equation 1, are

$$
\left.\begin{array}{l}
I_{z} \dot{\omega}_{z}-M_{z}^{\omega_{z}} \omega_{z}=\mu_{2} \delta_{2} R_{x}-\left(\mu_{1} \delta_{1}+\mu_{2} l_{2}+\mu_{3} l_{3}-l_{R}\right) R_{y}+M_{b z}+f_{z 1} \\
\dot{\alpha}=\omega_{z}+f_{z 2}
\end{array}\right\}
$$

Where, $M_{b z}$ represents RCS periodic equivalent torque with a period of $T_{d}, l_{R}$ is axial coordinate of press center in body frame, supposed to be a constant.

Let $M_{a z}=\mu_{2} \delta_{2} R_{x}-\left(\mu_{1} \delta_{1}+\mu_{2} l_{2}+\mu_{3} l_{3}-l_{R}\right) R_{y}$ be instantaneous MMC torque, $M_{c z}=M_{a z}+M_{b z}$ be compound control torque, and thus the control model for virtual controller design can be obtained according to Equation 3, which is

$$
I_{z} \ddot{\alpha}-M_{z}^{\omega_{z}} \dot{\alpha}=M_{c z}+f_{z}
$$

With full consideration of engineering practicability, PID control method is used to design the virtual controller. Therefore the control law is

$$
M_{c z}=K_{p} e_{\alpha}+K_{d} \dot{e}_{\alpha}+K_{i} \int_{0}^{t} e_{\alpha} d t
$$

Where $e_{\alpha}=\alpha_{r}-\alpha$ is attack angle error.

\subsection{Definition and Prediction of MMCPET}

We define MMCPET as the average torque of all instantaneous MMC torque working on aircraft during a period of $T_{d}$, which can be described as

$$
M_{a p}=\frac{1}{T_{d}} \int_{0}^{T_{d}}\left[\mu_{2} \delta_{2} R_{x}-\left(\mu_{1} \delta_{1}+\mu_{2} l_{2}+\mu_{3} l_{3}-l_{R}\right) R_{y}\right] d t
$$

Accordingly, only after all aerodynamic forces and MMC block position coordinates during the period are known, MMCPET is able to be calculated. However, to distribute the control torque, what we need is to predict MMCPET in the beginning of every period, and the exact information mentioned above cannot be known. To solve this problem, we use MMCS actuator models to predict MMC block position coordinates during the period, and simplify MMCPET calculation by taking aerodynamic forces as constant values in one period. As a result, the MMCPET prediction method we got is effective when following conditions are satisfied:

- $\quad$ The pulse-width modulation period $T_{d}$ is short enough that aerodynamic force changes during the period can be neglected reasonably. 
- $l_{R}$ and $\delta_{1}$ are constants, so as the distance between press center and total mass center $x_{P C}=\mu_{1} \delta_{1}+\mu_{2} l_{2}+\mu_{3} l_{3}-l_{R}$.

- At the beginning of every period, aircraft apparent acceleration $a_{A x}, a_{A y}$, position coordinate $\delta_{20}$ and relative velocity $\dot{\delta}_{20}$ of the second block can be easily measured on line. Thus the aerodynamic forces at that time can be calculated, and the equations are $R_{x}=m_{s} a_{A x}, R_{y}=m_{s} a_{A y}$.

- MMCS servo characteristic can be described as Equation 2, and values of $\xi_{2}, \omega_{2}$ are known. The space constraint condition of second block is $\left|\delta_{2}\right| \leq y_{\max }$

When above conditions are all satisfied, the prediction of MMCPET can be described as

$$
\tilde{M}_{a p}=-m_{s} a_{A y} x_{P C}+m_{s} a_{A x} \mu_{2}\left[\frac{1}{T_{d}} \int_{0}^{T_{d}} \delta_{2}(t) d t\right]
$$

Where, position coordinate prediction $\delta_{2}(t)$ of the second block during the period is

$$
\delta_{2}(t)=\delta_{2 c}-\frac{\dot{\delta}_{20}+\omega_{2}\left(\xi_{2}-\sqrt{\xi_{2}^{2}-1}\right)\left(\delta_{20}-\delta_{2 c}\right)}{2 \omega_{2} \sqrt{\xi_{2}^{2}-1} e^{\left(\xi_{2}+\sqrt{\xi_{2}^{2}-1}\right) \omega_{2} t}}+\frac{\dot{\delta}_{20}+\omega_{2}\left(\xi_{2}+\sqrt{\xi_{2}^{2}-1}\right)\left(\delta_{20}-\delta_{2 c}\right)}{2 \omega_{2} \sqrt{\xi_{2}^{2}-1} e^{\left(\xi_{2}-\sqrt{\xi_{2}^{2}-1}\right) \omega_{2} t}}, \quad\left|\delta_{2 c}\right| \leq y_{\max }
$$

Equations 7-8 show that MMCPET for one control period is single function of servo command $\delta_{2 c}$, because values of $a_{A x}, a_{A y}, \delta_{20}, \dot{\delta}_{20}$ are known constants during the period.

\subsection{Optimal Torque Distribution Rules and Optimal Solution}

To make full use of MMC torque, we define $J=\left(M_{c z}-\tilde{M}_{a p}\right)^{2} / 2$ as our optimal objective function for optimal torque distribution, which can be described as. Let $\delta_{2 c}^{*}$ be the optimal solution of the problem described by

$$
\min _{-y_{\max }<\delta_{2}<y_{\max }} J
$$

And the optimal torque distribution rules are

$$
\left.\begin{array}{l}
M_{a c}=\tilde{M}_{a p}\left(\delta_{2 c}^{*}\right) \\
M_{b c}=M_{c z}-\tilde{M}_{a p}\left(\delta_{2 c}^{*}\right)
\end{array}\right\}
$$

Where, the distributive MMC torque $M_{a c}$ equals to the optimal MMCPET $\tilde{M}_{a p}\left(\delta_{2 c}^{*}\right)$, and the distributive reaction control torque $M_{b c}$ is the compensation torque corresponding to the optimal MMCPET.

The above optimal problem is not difficult to be solved. Define two constants to simplify the optimal objective function, which are

$$
\left.\begin{array}{l}
a_{\delta}=-m_{s} a_{A x} \mu_{2}\left[1-\frac{2 \xi_{2}}{\omega_{2}}-\frac{2 \xi_{2}^{2}-1-2 \xi_{2} \sqrt{\xi_{2}^{2}-1}}{2 \omega_{2} \sqrt{\xi_{2}^{2}-1}} e^{-\left(\xi_{2}+\sqrt{\xi_{2}^{2}-1}\right) \omega_{2} T_{d}}+\frac{2 \xi_{2}^{2}-1+2 \xi_{2} \sqrt{\xi_{2}^{2}-1}}{2 \omega_{2} \sqrt{\xi_{2}^{2}-1}} e^{-\left(\xi_{2}-\sqrt{\xi_{2}^{2}-1}\right) \omega_{2} T_{d}}\right] \\
b_{\delta}=M_{c z}+m_{s} a_{A y} x_{P C}+m_{s} a_{A x}^{B} \mu_{2} \frac{\dot{\delta}_{20}+\delta_{20} \omega_{2}\left(\xi_{2}-\sqrt{\xi_{2}^{2}-1}\right)}{2 \omega_{2}^{2} \sqrt{\xi_{2}^{2}-1}\left(\xi_{2}+\sqrt{\xi_{2}^{2}-1}\right)}\left[e^{-\left(\xi_{2}+\sqrt{\xi_{2}^{2}-1}\right) \omega_{2} T_{d}}-1\right]+m_{s} a_{A x}^{B} \mu_{2} \frac{\dot{\delta}_{20}+\delta_{20} \omega_{2}\left(\xi_{2}+\sqrt{\xi_{2}^{2}-1}\right)}{2 \omega_{2}^{2} \sqrt{\xi_{2}^{2}-1}\left(\xi_{2}-\sqrt{\xi_{2}^{2}-1}\right)}\left[e^{-\left(\xi_{2}-\sqrt{\xi_{2}^{2}-1}\right) \omega_{2} T_{d}}-1\right]
\end{array}\right\}
$$

Accordingly, $J$ can be expressed as a quadratic polynomial function of $\delta_{2 c}$, as is shown below

$$
J=\left(a_{\delta} \delta_{2 c}+b_{\delta}\right)^{2} / 2
$$

From Equation 12, we can obtain the nonrestraint solution $\delta^{*}=-b_{\delta} / a_{\delta}$, which satisfies $\left[\partial J / \partial \delta_{2 c}\right]_{\delta^{*}}=0$. Define $\boldsymbol{Y}_{c}$ as the feasible set of the optimal problem, which can be described as $\boldsymbol{Y}_{c}=\left\{-y_{\max }, y_{\max }\right\} \cup \boldsymbol{Y}_{\boldsymbol{J}}$. Here, if $-y_{\max }<\delta^{*}<y_{\max }, \quad \boldsymbol{Y}_{\boldsymbol{J}}=\left\{\delta^{*}\right\}$, otherwise $\boldsymbol{Y}_{\boldsymbol{J}}=\varnothing$. By searching within $\boldsymbol{Y}_{\boldsymbol{c}}$, we can easily obtain the optimal solution $\delta_{2 c}^{*}$.

\subsection{Servo Commands of MMCS and RCS}

After we solve the optimal torque distribution problem, the distributive torque for MMCS and RCS are obtained. Then we can calculate the servo commands for these two control systems. For MMCS, servo command is 
position coordinate command of second block. To realize optimal MMCPET, MMC command is chosen to be the optimal solution $\delta_{2 c}^{*}$. As for RCS, servo command is actual RCS thrust commands for pitch thrusters. When expected RCS torque $M_{b c}$ is known, RCS thrust commands are able to be calculated by pulse width modulation rules.

\section{Simulations Results and Analysis}

To validate the proposed compound control method, we perform a group of comparison simulations, and the simulation results are shown in Figures 3-5.

With same object, attitude control requirement and virtual controller parameters, performances of compound control method, moving mass control method and reaction control method are compared. In the simulations, MMCS parameters are set to $y_{\max }=0.4, \xi_{2}=0.707, \omega_{2}=30 ;$ RCS parameters are set to $P_{e 1}=1000, P_{e 2}=100, T_{d}=15 \mathrm{~ms}$; other parameters are set to $H=30 \mathrm{~km}, V=5000 \mathrm{~m} / \mathrm{s}$. The numerical criterion of high performance is set to be realizing a rising time less than 1 second.

According to simulation results, only MMC is deficiency to realize the expected rising time, and the rising time of compound control is even less than that of reaction control. At the same time, comparing compound control with reaction control, with nearly the same control performance, the thrust impulse requirement ratio from the former to the latter is about 1:5. Therefore, the proposed compound control is better than moving mass control and reaction control, and is able to provide high performance with low energy cost for whole airspace reentry aircrafts.

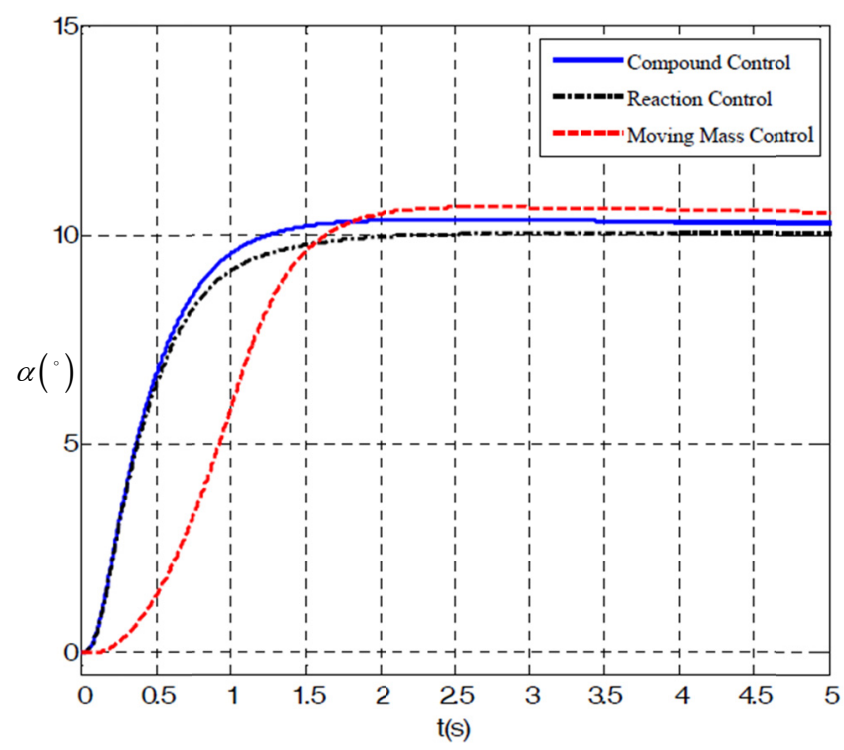

Figure 3. Attack angle response comparison 


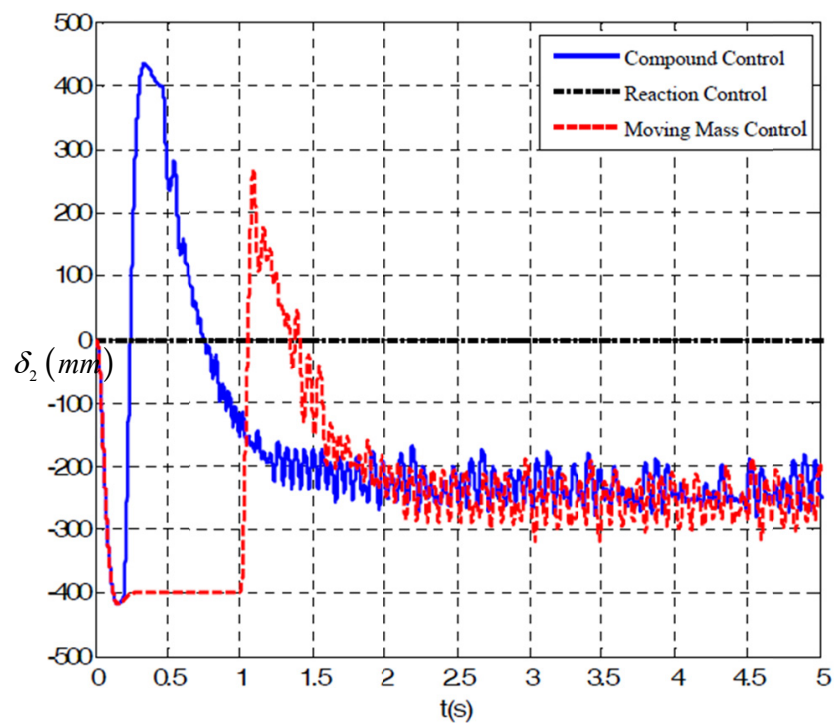

Figure 4. Second movable block coordinate comparison

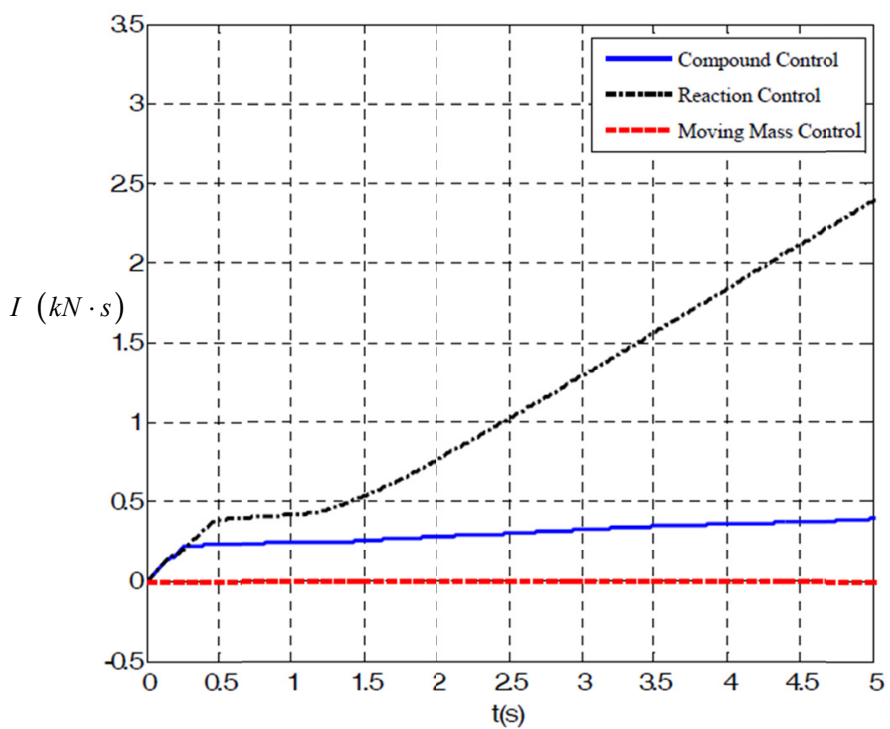

Figure 5. Reaction thrust impulse comparison

\section{Conclusions}

In this paper, we focus on combining moving mass control with reaction control to obtain a compound control for whole airspace reentry aircrafts, in order to achieve control system design target of high performance and low energy cost. An optimal torque distribution compound control system design is proposed and comparison simulation results demonstrate its validity. Moreover, the definition of moving mass control periodic equivalent torque (MMCPET) is the first time put forward, and the optimal torque distribution method based on MMCPET prediction is a helpful exploration to moving mass and reaction compound control system design.

\section{Acknowledgements}

Thanks and gratitude to Institute of Precise Guidance and Control in Northwestern Polytechnical University for the support given in making this study successful.

\section{References}

Guo, Q., Qin, K. Y., \& Yang, M. (2010). Integrated guidance and control method of mass moment combined control interceptor. Journal of Solid Rocket Technology, 33(4), 363-368.

Kong, X., Yang, M., \& Wang, S. Y. (2009). Attitude Control of Mass Torque and Lateral Thrust Combined Control Flight Vehicle. Computer Simulation, 26(5), 52-56. 
Lin, P., Zhou, F. Q., \& Zhou, J. (2007). Moving Centroid Control Mode for Reentry Warhead. Aerospace Control, 25(2), 16-20.

Lin, P., Zhou, J., \& Zhou, F. Q. (2009). Maneuverability Analysis of Reentry Vehicles Based on Moving Centroid Control Mode. Aerospace Control, 27(1), 7-9.

Menon, P. K., Sweriduk, G., Ohlmeyer, E. J., \& Malyevac, D. S. (2004). Integrated Guidance and Control of Moving Mass Actuated Kinetic Warheads. Journal of Guidance Control and Dynamics, 27(1), 118-126. http://dx.doi.org/10.2514/1.9336

Petsopoulos, T., Regan, F., \& Barlow, J. (1996). Moving-Mass Roll Control System for Fixed-Trim Re-Entry Vehicle. Journal of Spacecraft and Rockets, 33(1), 54-60. http://dx.doi.org/10.2514/3.55707

Robinett III, R. D., Sturgis, B. R., \& Kerr, S. A. (1996). Moving mass trim control for aerospace vehicles. Journal of Guidance, Control, and Dynamics, 19(5), 1064-1070. http://dx.doi.org/10.2514/3.21746

Wang, J. S., Xu, S. H., \& Liu, H. B. (2006). Design and Simulation of Distribution Strategy of Compound Control in Flying Saucer. Tactical Missile Technology, 3, 69-73. 